\title{
Integrated analysis of the conformation of a DNA/RNA- linked spin label by combining NMR ensembles and molecular dynamics simulations provides more realistic models of DNA/RNA structures in gum cancer cells using optimization of NMR spectroscopy of encapsulated DNA/ RNA dissolved in gum cancer cells
}

\author{
Alireza Heidari $^{1,2 *}$ and Ricardo Gobato ${ }^{3}$ \\ ${ }^{1}$ Faculty of Chemistry, California South University, 14731 Comet St. Irvine, CA 92604, USA \\ ${ }^{2}$ American International Standards Institute, Irvine, CA 3800, USA \\ ${ }^{3}$ Green Land Landscaping and Gardening, Seedling Growth Laboratory, 86130-000, Parana, Brazil
}

\begin{abstract}
In the current paper, integrated analysis of the conformation of a DNA/RNA-linked spin label by combining NMR ensembles and molecular dynamics simulations provides more realistic models of DNA/RNA structures in gum cancer cell using optimization of NMR spectroscopy of encapsulated DNA/RNA dissolved in gum cancer cells are studied. Gum cancer is a disease, which consists of division of abnormal cells without control. Blood and lymph systems can help cancerous cells spread to other parts of the body. Integrated analysis of the conformation of a DNA/RNA-linked spin label by combining NMR ensembles and molecular dynamics simulations provides more realistic models of DNA/RNA structures in gum cancer cells using optimization of NMR spectroscopy of encapsulated DNA/RNA dissolved in gum cancer cells is the most common of all gum cancer diagnosis and treatment techniques and methods. Screening for gum cancer is usually made by visual inspection of the lesions. Due to its subjective nature, the clinical accuracy of this screening can range from good to poor depending on the experience of the clinician. It is desirable to replace this subjective process with a non-invasive, reliable, simple, and integrated analysis of the conformation of a DNA/RNA-linked spin label by combining NMR ensembles and molecular dynamics simulations provides more realistic models of DNA/RNA structures in gum cancer cells using optimization of NMR spectroscopy of encapsulated DNA/RNA dissolved in gum cancer cells with high sensitivity and specificity. There is a high chance of cure for gum cancer in its early stages. Examinations are essential due to this reason.
\end{abstract}

\section{Introduction}

Cells interacting with their environment receive signals that are transduced to the nucleus through a complex array of biochemical signals known as signal transduction processes. These signaling events are specific for the agonist/s but can have some common features, like the activation of phosphorylation/dephosphorylation processes on a specific set of macromolecules. Biochemical, functional, structural and dynamical changes occurring also in complex systems subjected to specific perturbations can be identified by integrated analysis of the conformation of a DNA/RNA-linked spin label by combining NMR ensembles and molecular dynamics simulations provides more realistic models of DNA/RNA structures in gum cancer cells using optimization of NMR spectroscopy of encapsulated DNA/RNA dissolved in gum cancer cells. For instance, during typical time-course or dose-response experiments with drugs, the spectral features from groups that do not change during the stimulation annihilated one another, and only the changes occurring as a consequence of the activation/inhibition become evident in the difference spectrum. integrated analysis of the conformation of a DNA/RNA-linked spin label by combining NMR ensembles and molecular dynamics simulations provides more realistic models of DNA/RNA structures in gum cancer cells using optimization of NMR spectroscopy of encapsulated DNA/RNA dissolved in gum cancer cells is rapidly emerging as a bioanalytical tool for gum cancer diagnosis and therapy. The integrated analysis of the conformation of a DNA/RNA-linked spin label by combining NMR ensembles and molecular dynamics simulations provides more realistic models of DNA/RNA structures in gum cancer cells using optimization of NMR spectroscopy of encapsulated DNA/RNA dissolved in gum cancer cells molecular diagnostics have progressed from proof-of-concept

${ }^{\star}$ Correspondence to: Alireza Heidari, Faculty of Chemistry, California South University, 14731 Comet St. Irvine, CA 92604; American International Standards Institute, Irvine, CA 3800, USA, E-mail: Scholar.Researcher.Scientist@gmail. com; Alireza.Heidari@calsu.us; Central@aisi-usa.org

Key words: integrated analysis, conformation, DNA/RNA, linked spin label, nmr spectroscopy, molecular dynamics, simulations, realistic models, gum cancer cells, optimization

Received: February 23, 2021; Accepted: March 15, 2021; Published: March 19, 2021 
Heidari A (2021) Integrated analysis of the conformation of a DNA/RNA-linked spin label by combining NMR ensembles and molecular dynamics simulations provides more realistic models of DNA/RNA structures in gum cancer cells using optimization of NMR spectroscopy of encapsulated DNA/RNA dissolved in gum cancer cells

studies towards analysis in animal models as well as for in vitro clinical diagnostics in the last decade. Recently, integrated analysis of the conformation of a DNA/RNA-linked spin label by combining NMR ensembles and molecular dynamics simulations provides more realistic models of DNA/RNA structures in gum cancer cells using optimization of NMR spectroscopy of encapsulated DNA/RNA dissolved in gum cancer cells has also been implemented in screening, diagnosis, and staging of clinical gum cancer samples. Moreover, in vivo integrated analysis of the conformation of a DNA/RNA-linked spin label by combining NMR ensembles and molecular dynamics simulations provides more realistic models of DNA/RNA structures in gum cancer cells using optimization of NMR spectroscopy of encapsulated DNA/ RNA dissolved in gum cancer cells imaging has been implemented for mapping the extent of tumor growth and metastasis; integrated analysis of the conformation of a DNA/RNA-linked spin label by combining NMR ensembles and molecular dynamics simulations provides more realistic models of DNA/RNA structures in gum cancer cells using optimization of NMR spectroscopy of encapsulated DNA/RNA dissolved in gum cancer cells nanoparticles have also enabled image-guided therapies strongly indicating integrated analysis of the conformation of a DNA/ RNA-linked spin label by combining NMR ensembles and molecular dynamics simulations provides more realistic models of DNA/RNA structures in gum cancer cells using optimization of NMR spectroscopy of encapsulated DNA/RNA dissolved in gum cancer cells technology can significantly complement the practice of oncology. Despite the progress, widespread clinical translation of integrated analysis of the conformation of a DNA/RNA-linked spin label by combining NMR ensembles and molecular dynamics simulations provides more realistic models of DNA/RNA structures in gum cancer cells using optimization of NMR spectroscopy of encapsulated DNA/RNA dissolved in gum cancer cells nanoparticles is still challenging. Current integrated analysis of the conformation of a DNA/RNA-linked spin label by combining NMR ensembles and molecular dynamics simulations provides more realistic models of DNA/RNA structures in gum cancer cells using optimization of NMR spectroscopy of encapsulated DNA/RNA dissolved in gum cancer cells strategies in diagnostic oncology require further development and standardization to progress from bench-top to point-of-care applications. The present review critically analyzes the current state of the art about various strategies for integrated analysis of the conformation of a DNA/RNA-linked spin label by combining NMR ensembles and molecular dynamics simulations provides more realistic models of DNA/RNA structures in gum cancer cells using optimization of NMR spectroscopy of encapsulated DNA/RNA dissolved in gum cancer cells-based gum cancer detection and staging from cellular metabolites, exosomes, circulating tumor cells, extracellular fluids, and gum cancer cells [1-10].

\section{Results and discussion}

Based on the experimental procedures and the mathematical modeling method explained before, the results of experiments and further mathematical analysis on the results are presented. Then the experimental results of healthy and cancerous subjects are compared. Globally, gum cancer is one of the leading causes of death, so the development of strategies for an early diagnosis of gum cancer is of great importance. Biochemical alterations precede morphological changes in cells and tissues, so studying gum cancer metabolome seems like a reasonable approach for early diagnosis, prognosis and to follow treatment progression. Integrated analysis of the conformation of a DNA/RNA-linked spin label by combining NMR ensembles and molecular dynamics simulations provides more realistic models of
DNA/RNA structures in gum cancer cells using optimization of NMR spectroscopy of encapsulated DNA/RNA dissolved in gum cancer cells is a valuable tool for studying the metabolome of biological samples, such as gum cancer cell lines. Unlike staining procedures and other histopathologic approaches, this technique is rapid, non-destructive and does not require reagents. The spectral differences that result from probing the biochemical composition of gum cancer and normal cells are indicative of distinct metabolic profiles, which allow the discrimination of different cells. Using integrated analysis of the conformation of a DNA/RNA-linked spin label by combining NMR ensembles and molecular dynamics simulations provides more realistic models of DNA/RNA structures in gum cancer cells using optimization of NMR spectroscopy of encapsulated DNA/RNA dissolved in gum cancer cells and multivariate statistical analysis, several alterations concerning the content of lipids, proteins, nucleic acids and carbohydrates have been identified in gum cancer cells, some of which can be regarded as potential biomarkers. This review focuses on FTIR integrated analysis of the conformation of a DNA/RNA-linked spin label by combining NMR ensembles and molecular dynamics simulations provides more realistic models of DNA/RNA structures in gum cancer cells using optimization of NMR spectroscopy of encapsulated DNA/RNA dissolved in gum cancer cells as a metabolomics tool to study and characterize gum cancer cell lines (Figure 1).

\section{Conclusion}

By exploiting integrated analysis of the conformation of a DNA/ RNA-linked spin label by combining NMR ensembles and molecular dynamics simulations provides more realistic models of DNA/RNA structures in gum cancer cells using optimization of NMR spectroscopy of encapsulated DNA/RNA dissolved in gum cancer cells, the brightness of the photon flux density reaching the sample is no more a limitation in the illumination of microscopic sample features. High quality spectra of individual cells with a diameter around $15 \mu \mathrm{m}$, which is the average diameter of a granulocyte, were obtained at beamline B22. For reference, samples composed of very homogeneous cell populations were measured and usable spectra with acceptable $S / R$ values were achieved with conventional source from larger areas (e.g. $50 \mu \mathrm{m})$,

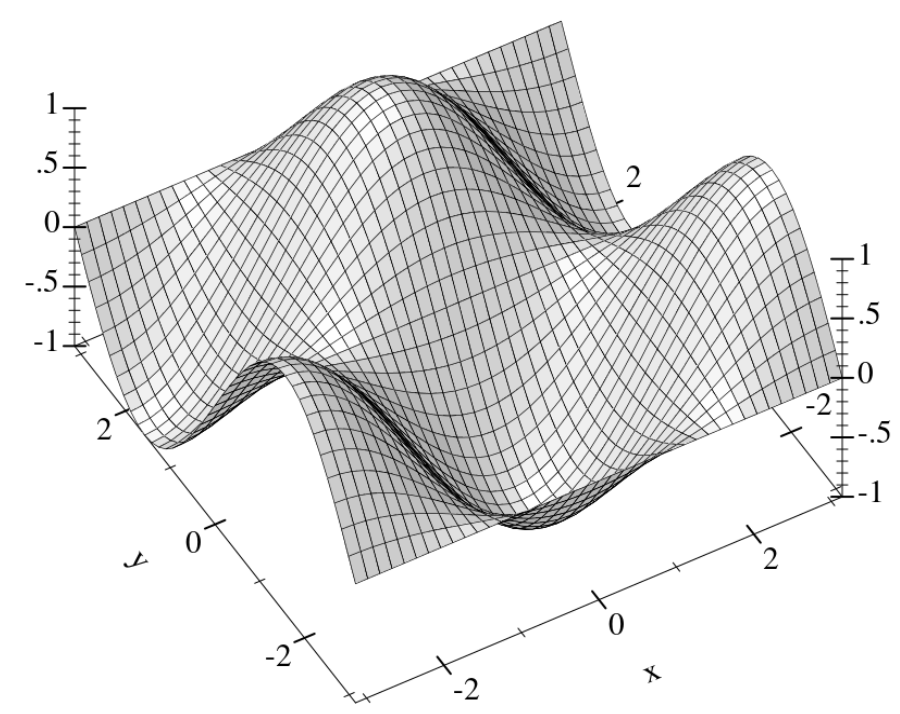

Figure 1. Integrated analysis of the conformation of a DNA/RNA-linked spin label by combining NMR ensembles and molecular dynamics simulations provides more realistic models of DNA/RNA structures in gum cancer cells using optimization of NMR spectroscopy of encapsulated DNA/RNA dissolved in gum cancer cells 
Heidari A (2021) Integrated analysis of the conformation of a DNA/RNA-linked spin label by combining NMR ensembles and molecular dynamics simulations provides more realistic models of DNA/RNA structures in gum cancer cells using optimization of NMR spectroscopy of encapsulated DNA/RNA dissolved in gum cancer cells

which demonstrated the complementarity of integrated analysis of the conformation of a DNA/RNA-linked spin label by combining NMR ensembles and molecular dynamics simulations provides more realistic models of DNA/RNA structures in gum cancer cells using optimization of NMR spectroscopy of encapsulated DNA/RNA dissolved in gum cancer cells with conventional lab instruments.

\section{Acknowledgments}

This study was supported by the Cancer Research Institute (CRI) Project of Scientific Instrument and Equipment Development, the National Natural Science Foundation of the United Sates, the International Joint BioSpectroscopy Core Research Laboratory Program supported by the California South University (CSU), and the Key project supported by the American International Standards Institute (AISI), Irvine, California, USA.

\section{References}

1. Heidari, C. Brown, "Study of Composition and Morphology of Cadmium Oxide (CdO) Nanoparticles for Eliminating Cancer Cells”, J Nanomed Res., Volume 2, Issue 5, 20 Pages, 2015.

2. A. Heidari, C. Brown, "Study of Surface Morphological, Phytochemical and Structural Characteristics of Rhodium (III) Oxide ( $\mathrm{Rh}_{2} \mathrm{O}_{3}$ ) Nanoparticles", International Journal of Pharmacology, Phytochemistry and Ethnomedicine, Volume 1, Issue 1, Pages 15-19, 2015.
3. A. Heidari, "An Experimental Biospectroscopic Study on Seminal Plasma in Determination of Semen Quality for Evaluation of Male Infertility", Int J Adv Technol 7: e007, 2016.

4. A. Heidari, "Extraction and Preconcentration of N-Tolyl-Sulfonyl-PhosphoramidSaeure-Dichlorid as an Anti-Cancer Drug from Plants: A Pharmacognosy Study", J Pharmacogn Nat Prod 2: e103, 2016.

5. A. Heidari, "A Thermodynamic Study on Hydration and Dehydration of DNA and RNA-Amphiphile Complexes”, J Bioeng Biomed Sci S: 006, 2016.

6. A. Heidari, "Computational Studies on Molecular Structures and Carbonyl and Ketene Groups' Effects of Singlet and Triplet Energies of Azidoketene $O=C=C H-N N N$ and Isocyanatoketene $O=C=C H-N=C=O$ ", J Appl Computat Math 5: e142, 2016.

7. A. Heidari, "Study of Irradiations to Enhance the Induces the Dissociation of Hydrogen Bonds between Peptide Chains and Transition from Helix Structure to Random Coil Structure Using ATR-FTIR, Raman and 'HNMR Spectroscopies", J Biomol Res Ther 5: e146, 2016.

8. A. Heidari, "Future Prospects of Point Fluorescence Spectroscopy, Fluorescence Imaging and Fluorescence Endoscopy in Photodynamic Therapy (PDT) for Cancer Cells”, J Bioanal Biomed 8: e135, 2016.

9. A. Heidari, "A Bio-Spectroscopic Study of DNA Density and Color Role as Determining Factor for Absorbed Irradiation in Cancer Cells", Adv Cancer Prev 1: e102, 2016.

10. A. Heidari, "Manufacturing Process of Solar Cells Using Cadmium Oxide (CdO) and Rhodium (III) Oxide (Rh, $O_{3}$ ) Nanoparticles", J Biotechnol Biomater 6: e125, 2016.

Copyright: (C2021 Heidari A. This is an open-access article distributed under the terms of the Creative Commons Attribution License, which permits unrestricted use, distribution, and reproduction in any medium, provided the original author and source are credited. 